\title{
Fluorescent In Situ Hybridization Protocols in Drosophila Embryos and Tissues
}

\author{
Eric Lécuyer, Neela Parthasarathy, and Henry M. Krause
}

\begin{abstract}
Summary
Fluorescent in situ hybridization is the standard method for visualizing the spatial distribution of RNA. Although traditional histochemical RNA detection methods suffered from limitations in resolution or sensitivity, the recent development of peroxidase-mediated tyramide signal amplification provides strikingly enhanced sensitivity and subcellular resolution. In this chapter, we describe optimized fluorescent in situ hybridization protocols for Drosophila embryos and tissues utilizing tyramide signal amplification, either for single genes or in a high-throughput format, which greatly increases the sensitivity, consistency, economy, and throughput of the procedure. We also describe variations of the method for RNA-RNA and RNA-protein codetection.
\end{abstract}

Key Words: Drosophila melanogaster; embryos and tissues; FISH; fluorescent in situ hybridization; RNA-protein costaining; single and double labeling; tyramide signal amplification.

\section{Introduction}

In situ hybridization in fixed tissues is the main method used for analyzing the spatial distribution of RNA, enabling the visualization of broad gene expression patterns, as well as subcellular localization properties (1). The method involves the recognition of the target RNA in situ through hybridization of a labeled antisense RNA probe. The most common detection strategy has been the use of digoxigenin (DIG)-labeled probes recognized by antibodies coupled to enzymes such as alkaline phosphatase (AP), which react with chromogenic substrates in order to reveal the distribution of the target RNA (2). Although this approach utilizes enzymatic amplification to increase staining sensitivity, the diffusibility of AP-generated dyes limits the resolution of the technique. Alternatively, the use of fluorescent in situ hybridization (FISH) presents several advantages, including the capacity to obtain clear views through thick samples, to reconstruct

From: Methods in Molecular Biology: Drosophila: Methods and Protocols

Edited by: C. Dahmann (C Humana Press Inc., Totowa, NJ 
three-dimensional images using high-resolution microscopy techniques, and the ability to compare multiple overlapping signals with high resolution. Conventional FISH uses fluorochrome-conjugated probe labels or antibodies that provide nondiffusible signals $(3,4)$, but are less sensitive as they lack an enzymatic signal amplification step. However, the use of tyramide signal amplification (TSA), involving the peroxidase-dependent complexing of fluorochromeconjugated tyramides to molecules in the vicinity of the probe, provides a strong enzymatically amplified signal and strikingly improved subcellular resolution (5-7).

This chapter describes our optimized procedures for performing high-resolution FISH on Drosophila embryos and dissected tissues, either for a few genes or in a high-throughput format in 96-well microtiter plates. Instructions are given for the preparation of RNA probes, the fixation of embryos and tissues, and the hybridization and TSA-mediated detection of probes. Also described are variations of the procedure for RNA-RNA and RNA-protein costaining. These protocols have been optimized for economy, high throughput, consistency, and sensitivity.

\section{Materials}

\subsection{RNA Probe Preparation (see Note 1)}

1. 1.5-mL Microcentrifuge tubes or standard 96-well V-bottom microplates.

2. RNAse-free water.

3. T7, T3, or SP6 RNA polymerase (Fermentas Life Sciences, Burlington, ON, Canada; cat. nos. EP0101, EP0111, and EP0131) as appropriate.

4. 10X Transcription buffer (supplied with polymerases): $0.4 M$ Tris-HCl, $\mathrm{pH} \mathrm{8.0,60}$ $\mathrm{mM} \mathrm{MgCl}{ }_{2}, 100 \mathrm{~m} M$ dithiothreitol, and $20 \mathrm{~m} M$ spermidine.

5. DIG RNA-labeling Mix (Roche Applied Science, Laval, QC, Canada; cat. no. 11 277073 910). Recommended for single FISH.

6. Biotin RNA-labeling mix (Roche Applied Science; cat. no. 11685597 910).

7. RNAguard (Amersham Biosciences, Piscataway, NJ; cat. no. 27-0816-01).

8. $3 M$ Sodium acetate.

9. Cold $100 \%$ ethanol.

10. Cold $70 \%$ ethanol.

\subsection{Embryo Collection and Fixation}

1. Chlorine bleach solution diluted 1:1 with water.

2. 20-mL Glass scintillation vials (Fisher Scientific Limited, Nepean, ON, Canada; cat. no. 03-337-15) or 1-L glass bottle.

3. $40 \%$ Formaldehyde solution (prepared on the day of fixing from paraformaldehyde): In scintillation vial, mix $0.92 \mathrm{~g}$ of paraformaldehyde in $2.5 \mathrm{~mL}$ of water containing $35 \mu \mathrm{L}$ of $1 N \mathrm{KOH}$. Dissolve the paraformaldehyde by carefully heating the solution on a stirring hot plate in a fume hood. Once the solution cools down, 
filter through a $0.45-\mu \mathrm{m}$ filter and store at $4^{\circ} \mathrm{C}$ until ready for use. Scale up the recipe if a larger volume is required (see Note 2).

4. 1X Phosphate-buffered saline (PBS) solution.

5. Heptane.

6. Methanol.

\subsection{Single FISH on Drosophila Embryos}

\subsubsection{Postfixation, Hybridization, and Posthybridization Washes}

1. 5-mL Polypropylene tubes, $1.5-\mathrm{mL}$ and $0.5-\mathrm{mL}$ microcentrifuge tubes, or $0.2-\mathrm{mL}$ half-skirted 96-well polymerase chain reaction (PCR) plates (Abgene, Rochester, NY; cat. no. AB-0900).

2. Microplate sealing foil (Ultident, Saint-Laurent, QC, Canada; cat. no. 24-PCRAS-200).

3. PBT solution: $1 \mathrm{X}$ PBS and $0.1 \%$ Tween-20.

4. $40 \%$ Formaldehyde solution, freshly prepared (see Subheading 2.2.).

5. Proteinase K (20 mg/mL) (Sigma Aldrich, Oakville, ON, Canada; cat. no. P2308). Dissolve in double-distilled water and store aliquots $(25-50 \mu \mathrm{L})$ at $-20^{\circ} \mathrm{C}$.

6. Glycine solution: $2 \mathrm{mg} / \mathrm{mL}$ glycine in PBT.

7. RNA hybridization solution: $50 \%$ formamide, $5 X$ SSC, $100 \mu \mathrm{g} / \mathrm{mL}$ heparin, $100 \mu \mathrm{g} / \mathrm{mL}$ sonicated salmon sperm DNA, and $0.1 \%$ Tween-20. Filter through a $0.2-\mu \mathrm{m}$ filter and store at $-20^{\circ} \mathrm{C}$ (stable for several months).

8. Heating block(s) or water bath(s) adjustable to 56,80 , and $100^{\circ} \mathrm{C}$, or $\mathrm{PCR}$ machine.

\subsubsection{Development of FISH Signal}

1. 1X PBS solution.

2. PBT solution: $1 \mathrm{X}$ PBS, $0.1 \%$ Tween-20.

3. PBTB solution: $1 \mathrm{X}$ PBS, $0.1 \%$ Tween-20, and $1 \%$ milk powder.

4. Detection of DIG-labeled probe.

a. Biotinylated anti-DIG antibody followed by streptavidin-HRP (Molecular Probes, Eugene OR), recommended to obtain strongest signal: biotin-conjugated mouse monoclonal anti-DIG (1/400 dilution of a $1 \mathrm{mg} / \mathrm{mL}$ stock solution in PBTB; Jackson ImmunoResearch Laboratories Inc., West Grove, PA; cat. no. 200-062-156) and streptavidin-HRP conjugate (1/100 dilution of a $1 \mu \mathrm{g} / \mathrm{mL}$ stock solution in PBTB; Molecular Probes; cat. no. S991).

b. HRP-conjugated anti-DIG antibodies, suitable for strongly expressed genes or for double-labeling experiments: HRP-conjugated mouse monoclonal anti-DIG (1/400 dilution of a $1 \mathrm{mg} / \mathrm{mL}$ stock solution in PBTB; Jackson ImmunoResearch Laboratories Inc.; cat. no. 200-032-156) or HRP-conjugated sheep monoclonal anti-DIG (1/500 dilution of stock solution in PBTB; Roche Applied Science; cat. no. 1207 733).

5. TSA: Cy3 tyramide conjugates (1/50 dilution of stock solution in amplification buffer; Perkin Elmer Life Sciences, Boston, MA; cat. no. SAT704A) or Alexa Fluor 488 tyramide conjugate (1/50 dilution of stock solution in amplification buffer; 
Molecular Probes; cat. no. T-20932). See Note 3 for advice on when to use the reagents described in steps $\mathbf{4}$ and $\mathbf{5}$.

6. $100 \mathrm{X} 4^{\prime}$,6-diamidino-2-phenylindole (DAPI) solution $\left(0.1 \mathrm{mg} / \mathrm{mL}\right.$ in $\left.\mathrm{H}_{2} \mathrm{O}\right)$.

\subsubsection{Storage, Mounting, and Viewing of Samples}

1. Mountant: 70\% glycerol, 2.5\% 1,4-diazabicyclo [2.2.2.] Octane (DABCO, Sigma Aldrich; cat. no. D-2522). In light-shielded tube, add $1.25 \mathrm{~g}$ of DABCO crystals, $15 \mathrm{~mL}$ of $1 \mathrm{X}$ PBS, and $35 \mathrm{~mL}$ of glycerol and mix on rocking platform until the solution is homogeneous. Store at $-20^{\circ} \mathrm{C}$.

2. Microscope slides.

3. Cover slips $\left(22 \times 22 \mathrm{~mm}^{2}\right)$.

4. Fluorescence or confocal microscope.

\subsection{Double FISH on Drosophila Embryos}

1. Reagents for postfixation of embryos, probe hybridization, and mounting of samples as described in Subheadings 2.3.1. and 2.3.3.

2. 1X PBS solution.

3. PBT solution: $1 \mathrm{X}$ PBS and $0.1 \%$ Tween- 20 .

4. PBTB solution: $1 \mathrm{X}$ PBS, $0.1 \%$ Tween-20, and $1 \%$ milk powder.

5. Quenching solution: $1 \mathrm{X}$ PBT and $1 \% \mathrm{H}_{2} \mathrm{O}_{2}$.

6. Detection of DIG-labeled probe with HRP-conjugated antibodies: HRP-conjugated mouse monoclonal anti-DIG (1/400 dilution of a $1 \mathrm{mg} / \mathrm{mL}$ stock solution in PBTB; Jackson ImmunoResearch Laboratories Inc.; cat. no. 200-032-156) or HRPconjugated sheep monoclonal anti-DIG (1/500 dilution of stock solution in PBTB; Roche Applied Science; cat. no. 1207 733).

7. Detection of biotin-labeled probe: streptavidin-HRP conjugate (1/100 dilution of a $1 \mu \mathrm{g} / \mathrm{mL}$ stock solution in PBTB; Molecular Probes; cat. no. S991).

8. TSA: Cy3 tyramide conjugate (1/50 dilution of stock solution in amplification buffer; Perkin Elmer Life Sciences; cat. no. SAT704A). Alexa Fluor 488 tyramide conjugate (1/50 dilution of stock solution in amplification buffer; Molecular Probes; cat. no. T-20932). See Note 3 for advice on when to use the reagents described in steps 6-8.

\subsection{RNA-Protein Double Labeling}

1. Reagents for postfixation of embryos, probe hybridization, detection of probes, and mounting of samples as described in Subheadings 2.3.1.-2.3.3.

2. Primary antibody directed against the protein of interest. To prevent antibody cross-detection, make sure that the species origin of this antibody differs from that of the anti-DIG antibody used to detect the FISH probe.

3. Select a fluorochrome-conjugated secondary antibody directed against the species of the primary antibody.

\subsection{FISH on Dissected Tissues}

1. 1.5-mL Microcentrifuge tubes.

2. 1X PBS solution. 
3. $40 \%$ Formaldehyde solution, freshly prepared (see Subheading 2.2.).

4. PBT solution: $1 \mathrm{X}$ PBS and $0.1 \%$ Tween-20.

5. Fixation solution: $1 \mathrm{X}$ PBS and $4 \%$ formaldehyde.

6. For single or double FISH, prepare reagents for probe hybridization and detection as described in Subheadings 2.3.1., 2.3.2., and/or 2.4.

\section{Methods}

\subsection{RNA Probe Preparation}

1. Different strategies can be used to prepare template DNA for synthesizing antisense RNA probes by in vitro transcription. A gene segment of interest should first be cloned into an appropriate plasmid containing flanking bacteriophage promoter sequences $(T 3, T 7$, or $S p 6)$. Then, the plasmid can either be linearized by restriction enzyme digestion or used as a template for PCR to generate an amplified gene fragment with promoter sequences on each extremity. The PCR based approach is particularly useful when templates for several genes are being prepared simultaneously, as most sequences can be amplified using universal primers that overlap the $T 7, S p 6$, and/or T3 sequences. Once the linearized DNA fragments or PCR products have been purified, either through traditional phenol/chloroform extraction combined with ethanol precipitation or agarose gel extraction, they can be used for in vitro transcription as detailed in step 2. Care should be taken to work in RNAse-free conditions. For most Drosophila genes, cDNA sequences cloned between flanking promoters are readily available in the Drosophila Gene Collections, and PCR protocols for the vectors used in these libraries have been described (8). For templates that are amplified in a 96-well plate format, the PCR products can be bulk purified by centrifugation using filter plates (Whatman Inc.; Clifton, NJ; cat. no. 7700-1303), concentrated by ethanol precipitation and centrifugation in V-bottom 96-well plates, and then resuspended in $15 \mu \mathrm{L}$ of RNAse-free water.

2. RNA probes are prepared as described on the product sheets of their DIG and biotin RNA-labeling kits (Roche Applied Science). On ice, mix 0.5-1 $\mu \mathrm{g}$ linearized template DNA or PCR product, $2 \mu \mathrm{L}$ DIG or biotin RNA-labeling mix, $2 \mu \mathrm{L} 10 \mathrm{X}$ transcription buffer, $1 \mu \mathrm{L}$ RNAguard ( $40 \mathrm{U} / \mu \mathrm{L}), 2 \mu \mathrm{L}$ RNA polymerase $(20 \mathrm{U} / \mu \mathrm{L})$, and RNAse-free water to a final volume of $20 \mu \mathrm{L}$. Incubate at $37^{\circ} \mathrm{C}$ for $2-4 \mathrm{~h}$.

For PCR templates amplified and purified in 96-well format, probes can be bulk synthesized in V-bottom microplates in a total reaction volume of $10 \mu \mathrm{L}$. In each well, $5 \mu \mathrm{L}$ of resuspended template is combined with $5 \mu \mathrm{L}$ of premixed and prealiquoted transcription reaction mixture containing: $1 \mu \mathrm{L} 10 \mathrm{X}$ transcription buffer, $0.5 \mu \mathrm{L}$ DIG-labeling mix, $0.4 \mu \mathrm{L}$ RNA polymerase $(20 \mathrm{U} / \mu \mathrm{L}), 0.125 \mu \mathrm{L}$ RNAguard $(40 \mathrm{U} / \mu \mathrm{L})$, and $3 \mu \mathrm{L}$ RNAse-free water. Plates are then sealed with adhesive foil and incubated for $2-4 \mathrm{~h}$ at $37^{\circ} \mathrm{C}$.

3. Once probe synthesis is completed, RNAse-free water is added to the reactions to bring the total volume up to $50 \mu \mathrm{L}$, then the probes are precipitated by adding 0.1 volumes $3 M$ sodium acetate and 2.5 volumes of cold $100 \%$ ethanol (see Note 4). Place at $-70^{\circ} \mathrm{C}$ overnight, then spin and wash the pellets with cold $70 \%$ ethanol. After drying, resuspend the probe pellets in $50 \mu \mathrm{L}$ RNAse-free water. Analyze and 
quantify the run-off transcripts through conventional agarose gel electrophoresis and ethidium bromide staining. Store probes at $-70^{\circ} \mathrm{C}$.

\subsection{Embryo Collection and Fixation}

The following steps can be performed on a small or large scale depending on the size of the fly chambers used for embryo collection.

1. Prepare $40 \%$ formaldehyde stock solution just before embryo dechorionation.

2. Collect and rinse embryos using room temperature tap water and a collection sieve.

3. Dechorionate the collected embryos in a chlorine bleach solution for approx $90 \mathrm{~s}$. As dechorionation proceeds, the embryos become clumpy and may tend to stick to the sides of the collection basket. Rinse the embryos immediately and thoroughly with fast flowing room temperature tap water or with embryo rinse solution $(0.7 \%$ $\mathrm{NaCl}$ and $0.03 \%$ Triton X-100) to remove residual bleach.

4. For small collections ( $<250 \mu \mathrm{L}$ settled embryos), transfer the embryos to a $20 \mathrm{~mL}$ glass scintillation vial containing a biphasic mixture of $8 \mathrm{~mL}$ heptane, $2 \mathrm{~mL}$ PBS, and $200 \mu \mathrm{L} 40 \%$ formaldehyde. For large collections ( $>5 \mathrm{~mL}$ of settled embryos), transfer embryos to a 1-L bottle containing $300 \mathrm{~mL}$ heptane, $90 \mathrm{~mL}$ PBS, and 10 $\mathrm{mL} 40 \%$ formaldehyde. Shake for $20 \mathrm{~min}$.

5. Using a Pasteur or serological pipet, eliminate the lower aqueous phase and most of the upper heptane phase, taking care not to draw up the embryos found at the interface. For small collections, transfer the embryos to a $1.5-\mathrm{mL}$ microfuge tube containing $0.5 \mathrm{~mL}$ heptane and $0.5 \mathrm{~mL}$ methanol. For large collections, transfer embryos to a $500-\mathrm{mL}$ bottle containing $100 \mathrm{~mL}$ heptane and $100 \mathrm{~mL}$ methanol. Devitellinize the embryos by shaking vigorously for $45 \mathrm{~s}$ until most of the embryos sink to the bottom. Carefully remove most of the heptane and add 1 or $100 \mathrm{~mL}$ of methanol, for small or large collections, respectively. Shake once more. All or most of the embryos should now sink to the bottom of the tube. Remove all of the liquid along with any unsettled embryos and rinse three times with methanol. Embryos can be pooled in polypropylene tubes and stored in methanol at $-20^{\circ} \mathrm{C}$ for several months.

\subsection{Single FISH on Drosophila Embryos}

\subsubsection{Postfixation, Hybridization, and Posthybridization Washes}

Hybridizations can be performed in $1.5 / 0.5-\mathrm{mL}$ microfuge tubes $(50 \mu \mathrm{L}$ settled embryos) or $0.2-\mathrm{mL}$ PCR plates $(10 \mu \mathrm{L}$ settled embryos/well). The latter are particularly well suited for optimizing experimental conditions (i.e., antibody titrations) or when many samples are processed in one experiment. Using the recommended PCR plates, which can easily be cut into smaller sections when processing a few dozen samples, greatly facilitates sample manipulation and long-term storage. Make sure to seal plates appropriately with sealing foil for all incubations and washes (see Note 5). Unless otherwise indicated, the wash volumes used below are $1 \mathrm{~mL}$ or $150 \mu \mathrm{L}$ for microfuge or PCR tubes, respectively. 
If not, the appropriate volumes for each tube format are provided, separated by or as above.

1. Aliquot embrïyos in tubes or plates (see Note 6).

2. Rinse the embryos once in methanol, once in a 1:1 mixture of methanol:PBT, and two times in PBT.

3. Postfix the embryos for 20 min in $4 \%$ formaldehyde (prepared by diluting fresh $40 \%$ formaldehyde 1/10 in PBT). Place tubes on a rocking platform or rotating mixer to ensure even fixation. If using PCR plates, secure plate in a vertical position to achieve more efficient mixing.

4. Wash embryos three times in PBT for 2 min each.

5. Prepare a working $3 \mu \mathrm{g} / \mathrm{mL}$ proteinase $\mathrm{K}$ solution from a $20 \mathrm{mg} / \mathrm{mL}$ stock by diluting in PBT. Add 500 or $100 \mu \mathrm{L}$ of proteinase K solution to each embryo sample and incubate at room temperature for $13 \mathrm{~min}$, or adjust the time according to the type of tissue (see Note 7). During this period, mix five to six times by gently rotating the tube once or twice or by jetting with a pipetman. Transfer the embryos to ice and incubate for $1 \mathrm{~h}$. This prolonged incubation on ice ensures uniform penetration and action of the protease.

6. Remove proteinase $\mathrm{K}$ solution and stop the digestion by performing a 2 min wash with a $2 \mathrm{mg} / \mathrm{mL}$ glycine solution with rocking. Repeat the glycine wash a second time.

7. Rinse embryos two times in PBT to remove the glycine.

8. Postfix the embryos again (as in step 3) for $20 \mathrm{~min}$ in $4 \%$ formaldehyde.

9. Wash embryos five times in PBT for 2 min each to remove all traces of fixative.

10. Rinse the embryos in a 1:1 mixture of PBT:RNA hybridization solution. Replace the mixture with $100 \%$ hybridization solution. At this point, the embryos can be stored for days/weeks at $-20^{\circ} \mathrm{C}$. If embryos were processed as a large batch (see Note 5), distribute embryos evenly into PCR plates using wide aperture tips, aiming for a final volume of $10 \mu \mathrm{L}$ settled embryos/well. If $1.5-\mathrm{mL}$ tubes were used up to this point, transfer embryos to $0.5-\mathrm{mL}$ tubes. When ready to hybridize, proceed to step 11.

11. In a separate tube, boil 400 or $100 \mu \mathrm{L} / \mathrm{sample}$ of RNA hybridization solution at $100^{\circ} \mathrm{C}$ for $5 \mathrm{~min}$, for 0.5 - or $0.2-\mathrm{mL}$ tubes, respectively. Cool on ice for at least 5 min. This freshly boiled hybridization solution will be used as the prehybridization solution.

12. Remove hybridization buffer from embryos. Add cooled prehybridization solution and place the embryos in a $56^{\circ} \mathrm{C}$ heat block/water bath. Incubate at $56^{\circ} \mathrm{C}$ for a minimum of $2 \mathrm{~h}$.

13. Prepare probe solution by adding $50-100 \mathrm{ng}$ of probe in $100 \mu \mathrm{L}$ of hybridization solution, heat at $80^{\circ} \mathrm{C}$ for $3 \mathrm{~min}$, and cool on ice for at least $5 \mathrm{~min}$. The probe solution can be kept on ice until prehybridization is completed.

14. Remove the prehybridization solution and add the probe solution to the embryos. Incubate at $56^{\circ} \mathrm{C}$ for $12-16 \mathrm{~h}$. This step is generally performed overnight.

15. Preheat all wash solutions to $56^{\circ} \mathrm{C}$. Remove the probe solution and rinse the embryos once with 400 or $100 \mu \mathrm{L}$ prewarmed hybridization buffer. Replace the rinse solution with another 400 or $100 \mu \mathrm{L}$ prewarmed hybridization buffer and incubate at $56^{\circ} \mathrm{C}$ for $15 \mathrm{~min}$. 
16. Wash for 15 min each with 400 or $100 \mu \mathrm{L}$ of $3: 1,1: 1$, and 1:3 mixtures of hybridization buffer:PBT.

17. Wash four times for 5 min each, with 400 or $100 \mu \mathrm{L}$ prewarmed PBT, then cool embryos to room temperature.

\subsubsection{Development of FISH Signal}

Unless otherwise indicated, the wash volumes used below are 400 or $150 \mu \mathrm{L}$ for $0.5-\mathrm{mL}$ tubes or $0.2-\mathrm{mL}$ PCR strips/plates, respectively. Antibody incubations and washes are performed in PBTB in order to reduce nonspecific staining (see Note 8).

1. Block embryos by incubating with PBTB for 10 min with constant mixing.

2. Incubate embryos with 300 or $100 \mu \mathrm{L}$ of the appropriate anti-DIG antibody solution for 2 h. (see Notes 3).

If an HRP-conjugated antibody is used in step 2, rinse embryos once with PBTB following the antibody incubation, then perform a nuclear counter stain by incubating for 10 min with a PBTB solution containing $1 \mathrm{X}$ DAPI, then proceed directly to step $\mathbf{6}$.

3. Perform six washes for $10 \mathrm{~min}$ each with PBTB.

4. Incubate embryos for $1 \mathrm{~h}$ with 200 or $75 \mu \mathrm{L}$ of streptavidin-HRP solution (diluted $1 / 100$ in PBTB).

5. Rinse embryos once with PBTB, then perform a nuclear counter stain by incubating for 10 min with a PBTB solution containing $1 X$ DAPI.

6. Wash the embryos six times for 10 min each with PBTB, then once with PBT and two times with PBS for 5 min each.

7. Prepare $1 / 50$ dilutions of the appropriate tyramide conjugate with the amplification buffer supplied in the tyramide kit (see Note 3). Remove the last PBS wash from the embryos, add 150 or $50 \mu \mathrm{L}$ tyramide solution, and incubate in the dark at room temperature for $2 \mathrm{~h}$ with constant mixing.

8. Wash six times for $10 \mathrm{~min}$ each with PBS.

\subsubsection{Storage, Mounting, and Viewing of Samples}

1. Resuspend embryos in 200 or $100 \mu \mathrm{L}$ of DABCO mountant. Allow the embryos to settle to the bottom of the tube $\left(1-3 \mathrm{~h}\right.$ or overnight at $4^{\circ} \mathrm{C}$ ) before mounting (see Note 9). Embryos can be stored for months/years in microfuge tubes or PCR plates at $4^{\circ} \mathrm{C}$ in light-shielded receptacles.

2. Transfer approx $35-\mu \mathrm{L}$ aliquot of embryos, by delicate resuspension using wide aperture tips, onto a clean slide and cover with a $22 \times 22 \mathrm{~mm}^{2}$ cover slip. Seal the edges with transparent nail polish. Slides can be stored for a few weeks at $4^{\circ} \mathrm{C}$ in the dark. In our experience, the DAPI stain tends to diffuse away after a few weeks on slides. Therefore, it is better to mount a fresh aliquot of embryos if samples are reanalyzed at a later date.

3. Analyze embryos by conventional fluorescence or confocal microscopy. 
The stainings shown in Fig. 1. exhibit mRNA expression/localization patterns-obtained FISH using TSA in comparison with conventional AP-based in situ hybridizations (images from BDGP: http://www.fruitfly.org/cgi-bin/ exinsitu.pl) (8).

\subsection{Double FISH on Drosophila Embryos}

1. Generate two probes, each with a different label, as described in Subheading 3.1. (see Note 1 for alternative/additional labels).

2. Collect and fix embryos as described in Subheading 3.2.

3. Perform the hybridization with both probes simultaneously; all other pre- and posthybridization washes are as described in Subheading 3.3.1.

4. Block embryos by incubating with PBTB for 10 min with constant mixing.

5. Incubate embryos with 300 or $100 \mu \mathrm{L}$ of the appropriate HRP-conjugated anti-DIG antibody solution for $2 \mathrm{~h}$. (see Note 3 ).

6. Wash the embryos six times for 10 min each with PBTB, then once with PBT and two times with PBS for 5 min each.

7. Prepare a 1/50 dilution of the first tyramide conjugate using the amplification buffer supplied in the tyramide kit (see Note 3). Remove the last PBS wash from the embryos, add 150 or $50 \mu \mathrm{L}$ tyramide solution, and incubate in the dark at room temperature for $2 \mathrm{~h}$ with constant mixing. All of the following steps should be carried out in a light-shielded receptacle.

8. Wash six times for $10 \mathrm{~min}$ each with PBS.

9. Quench the first tyramide reaction by washing for $15 \mathrm{~min}$ with quenching solution (see Note 10). Wash two times with PBS and two times with PBT for 5 min each.

10. Block embryos with PBTB for 10 min as in step 4.

11. Incubate embryos for $1 \mathrm{~h}$ with 200 or $75 \mu \mathrm{L}$ of streptavidin-HRP solution (diluted $1 / 100$ in PBTB).

12. Rinse embryos once with PBTB, then perform a nuclear counter stain by incubating for 10 min with a PBTB solution containing $1 X$ DAPI.

13. Wash the embryos six times for 10 min each with PBTB, then once with PBT and two times with PBS for 5 min each.

14. Prepare 1/50 dilutions of the second tyramide conjugate with the amplification buffer supplied in the tyramide kit (see Note 3 ). Add 150 or $50 \mu \mathrm{L}$ tyramide solution and incubate for $2 \mathrm{~h}$ with constant mixing.

15. Wash six times for 10 min each with PBS.

16. Mount and view samples as described in Subheading 3.3.3.

Figure 2 shows an example of a double FISH staining for mRNAs encoded by the CG1962 and Canoe genes.

\subsection{RNA-Protein Double Labeling}

1. Collect, process, and hybridize embryos essentially as described in Subheading 3.2. and 3.3.1.; with the exception of the proteinase K step, which may have to be adapted for optimal immunostaining (see Note 7). 

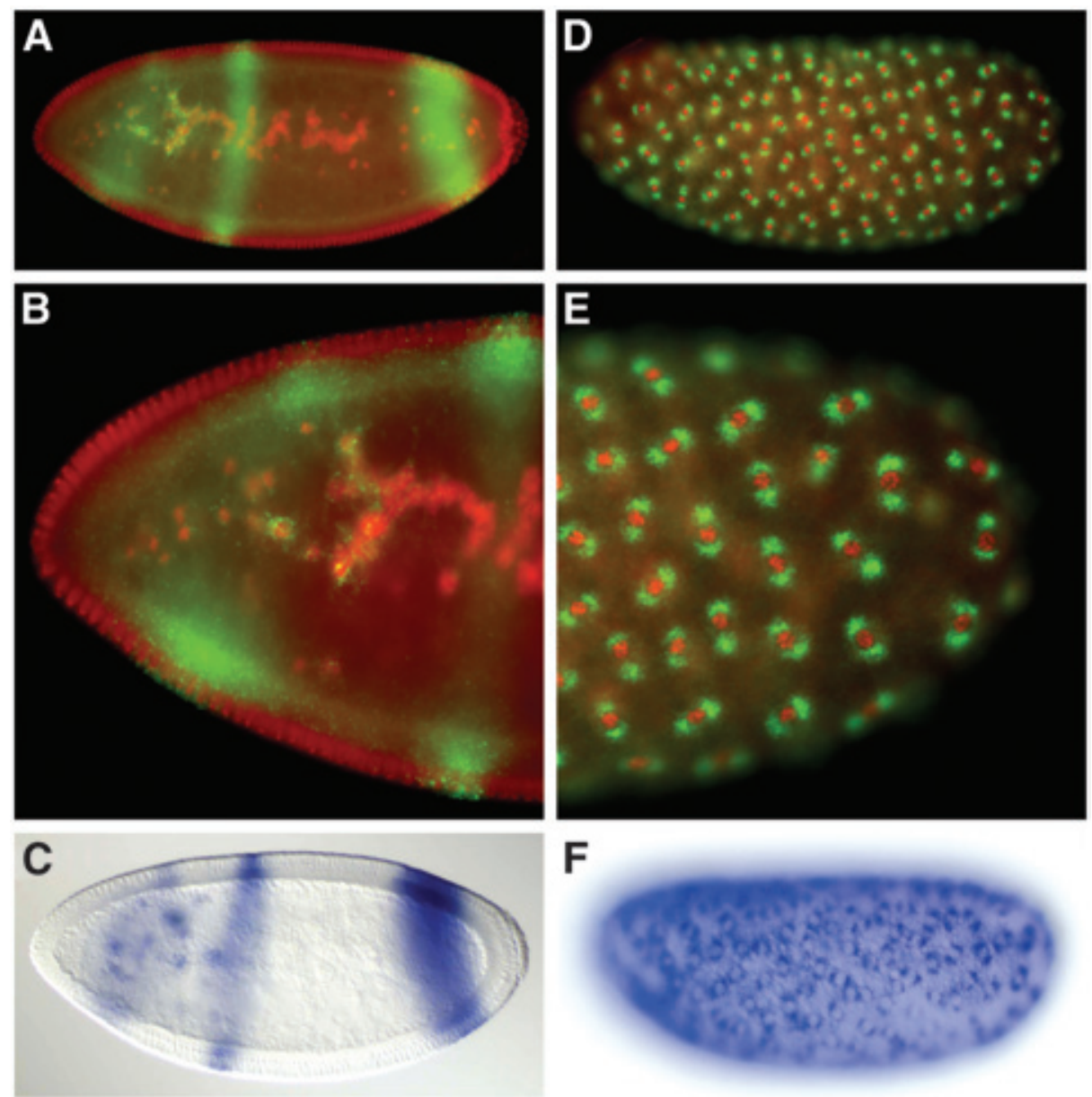

Fig. 1. Staining patterns obtained using DIG-labeled antisense probes for (A-C) Hunchback and (D-F) CG1962 transcripts, either visualized using TSA (A,B,D,E) or conventional AP-based detection (C,F). Tyramide stained embryos were processed through consecutive incubations with a biotinylated anti-DIG antibody, streptavidinHRP and Cy3 tyramide, whereas nuclei were counterstained using DAPI. The Cy3 tyramide and DAPI signals were false colored in green and red respectively, as this coloring scheme provides better contrast. (A-C) Zygotic Hunchback gene expression is detected in stripes of peripheral blastoderm nuclei and in a subset of yolk nuclei. Tyramide detection enables the visualization of nuclear foci representing nascent zygotic transcripts, as well as cytoplasmic mRNA pools. (D-F) Transcripts of the CG1962 gene demonstrate centrosome microtubule localization. AP-stained embryo images were obtained from the Berkeley Drosophila Genome Project in situ hybridization web resource (8). 

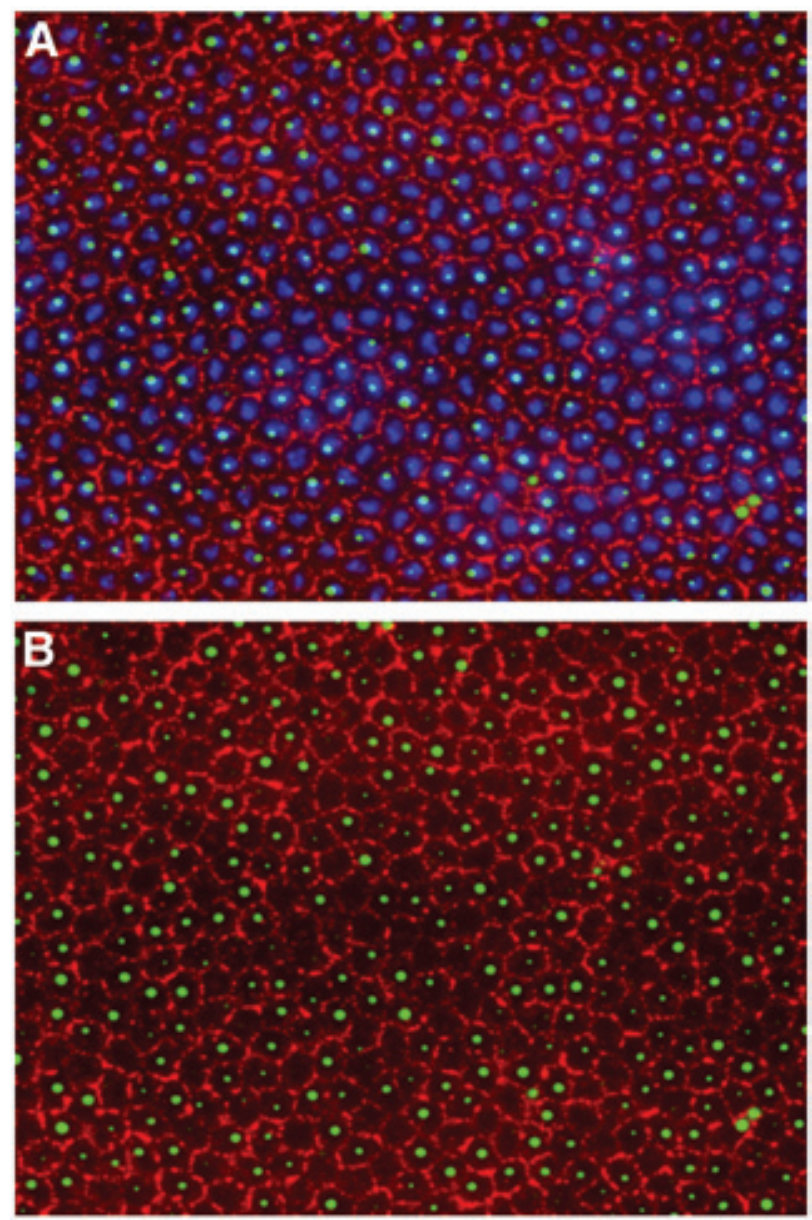

Fig. 2. Double FISH detection of CG1962 and Canoe gene transcripts. (A,B) Hybridizations were performed using a biotinylated probe for CG1962, detected with streptavidin-HRP and Alexa 488 tyramide (green signal); and a DIG-labeled probe for Canoe, revealed using an HRP-conjugated sheep anti-DIG antibody and Cy3 tyramide (red signal). The image shown in (A) also shows the nuclei counterstained with DAPI (blue signal). CG1962 mRNA localizes in foci that are localized above the nuclei, whereas Canoe gene transcripts are localized at membrane junctions that separated each nuclei.

2. Take care to select noncrossreactive detection reagents (i.e., antibodies generated in different host species). Add the primary antibody against the protein of interest, along with the appropriate probe detection reagent; HRP- or biotin-conjugated anti-DIG antibodies, or streptavidin-HRP, for DIG- and biotin-labeled probes, respectively. Incubate embryos with 300 or $100 \mu \mathrm{L}$ antibody solution (diluted in PBTB) for $2 \mathrm{~h}$ at room temperature with constant mixing. 
3. Perform six washes for 10 min each with PBTB.

4. Add secondary detection reagents (fluorochrome-conjugated secondary antibodies and streptavidin-HRP). Perform incubations, washes, DAPI staining, and TSA reaction as in Subheading 3.3.2. (see Note 11).

5. Mount samples as described in Subheading 3.3.3.

\subsection{FISH on Dissected Tissues}

1. Dissect tissues, such as imaginal discs and salivary glands, in 1X PBS. Dissected tissues can be stored briefly on ice in a $1.5-\mathrm{mL}$ microfuge tube containing PBS until enough tissue is obtained for analysis.

2. Remove the PBS and add $600 \mu \mathrm{L}$ of fixation solution. Shake gently for $20 \mathrm{~min}$.

3. Wash embryos five times in PBT for 2 min each to remove all traces of fixative.

4. Perform prehybridization, hybridization, antibody incubations, TSA reactions, and mounting of samples as described in Subheadings 3.3.1.-3.3.3.

\section{Notes}

1. The DIG and biotin labels described here can be substituted by or combined with many other labels, including fluorescein, dinitrophenyl, and a number of Alexaconjugated nucleotides. These can be detected by a variety of commercially available antibodies and provide numerous possibilities for multilabeling experiments, as described by Kosman et al. (7).

2. Preparing smaller batches of fresh formaldehyde solutions as needed ensures consistent and strong fixation of samples, whereas avoiding potential loss of activity that might occur with larger volumes of commercially available formaldehyde solutions kept in storage over long periods of time.

3. To obtain the strongest FISH signal, we recommend using the biotinylated anti-DIG antibody in combination with streptavidin-HRP, which provides an extra signal amplification step compared with the HRP-conjugated anti-DIG antibodies alone. However, although less sensitive, these directly conjugated antibodies are suitable for double FISH experiments, where biotin is used as a second probe label, or for RNA-protein codetection experiments, where antibody crossreactivity becomes a concern. Although we have mainly used Cy3 and Alexa 488 tyramide conjugates, which both give strong signals; there is a variety of additional fluor-conjugated tyramides available from Perkin Elmer Life Sciences and Molecular Probes. The amplification buffer supplied with the Perkin Elmer Life Sciences tyramide kits is in ready-to-use format. In contrast, when using Alexa tyramide conjugates from Molecular Probes, hydrogen peroxide supplied with the kit needs to be added to the amplification buffer $(0.0015 \%$ final concentration) before use. The recommended antibody and tyramide dilutions found to be optimal in our laboratory might need to be optimized on a lab-by-lab basis owing to variability in research environments and product stocks.

4. We have found that removal of DNA templates by DNAseI treatment following the transcription reactions, as well as carbonate degradation of probes for increased tissue penetration, to be unnecessary and may risk reducing probe quality. After performing side-by-side comparisons, we opted for using sodium acetate instead of lithium chloride for probe precipitation, as it provided greater precipitation efficiency. 
5. When intending to use PCR plates for hybridizations, it might be preferable to perform steps 1-10 in Subheading 3.3.1. using 5-mL polypropylene tubes containing approx $300 \mu \mathrm{L}$ settled embryos (one tube/quarter plate). This makes the manipulations easier at the proteinase $\mathrm{K}$ digestion step where delicate mixing is required and embryos can be aliquoted in PCR plates before starting the prehybridizations. When aliquoting the embryos into PCR plates, it is preferable to use a pipetman rather then a multichannel pipetor, as it is easier to maintain an even suspension of embryos by up and down pipeting in order to achieve equal embryo distribution in the plates. Take care to eliminate any air bubbles that may have formed under the embryo layer as these may damage the samples during the hybridization step. Once the embryos are aliquoted, multichannel pipetors are recommended for all subsequent washing, antibody incubation, and mounting steps. Furthermore, washes can be greatly facilitated by using an eight-well manifold connected to a vacuum pump to aspirate solutions.

6. When pipeting embryo/tissue samples, wide aperture tips should be used to avoid damaging the embryos. Wide aperture tips can be purchased from a variety of suppliers. If these are not available, simply cut off the ends of traditional tips.

7. Proteinase $\mathrm{K}$ digestion is an important parameter for optimal probe entry into the embryo or tissue of interest. Over digestion can disrupt tissue integrity and morphology, whereas under digestion can hinder even accessibility of the probe to the entire sample. Traditional protocols suggest a short incubation (1-5 min) at high proteinase $\mathrm{K}$ concentration $(50 \mu \mathrm{g} / \mathrm{mL})$; however, we have found that performing the digestions for a longer period of time at lower proteinase $\mathrm{K}$ concentrations, followed by a $1 \mathrm{~h}$ incubation on ice, significantly improves staining sensitivity and uniformity from embryo to embryo. When preparing new proteinase $\mathrm{K}$ stocks, or when working with new types of tissue (i.e., dissected tissues, mutant embryos that may be more delicate, and so on), we recommend titrating the concentration of proteinase $\mathrm{K}$ in order to find the optimal working concentration. Some tissues, such as dissected larval tissues, tend to be more sensitive to proteinase $\mathrm{K}$ digestion; as a result, we often omit the proteinase $\mathrm{K}$ step when dealing with such samples. It may also be necessary to reduce proteinase K levels when performing RNA-protein costaining experiments (see Subheading 3.5.), as over digestion may perturb epitope recognition.

8. The concentration of milk used in this protocol has been optimized for use with the antibodies described in Subheading 2.3.2. For other antibodies, it may be preferable to vary the concentration of milk or use alternate blocking reagents (i.e., bovine serum albumin or commercially available blocking solutions) to increase signal specificity.

9. We find that samples that have been precociously mounted often exhibit a hazy background appearance that dissipates a few hours after the mountant solution has been added to the embryos.

10. Although we have found quenching with $1 \%$ hydrogen peroxide to be satisfactory when performing double tyramide reactions, treatment with $0.01 \mathrm{M} \mathrm{HCl}$ for $10 \mathrm{~min}$ or heating at $70^{\circ} \mathrm{C}$ for 15 min have been suggested as alternative treatments for inactivating the first HRP reaction $(6,9)$. 
11. For RNA-protein costaining experiments; we have traditionally used secondary antibodies that are directly conjugated to a fluorogenic compound of interest. However, we have begun using TSA as a means of enhancing our immunostaining signal, through the use of HRP-conjugated secondary antibodies directed against the species of the primary antibody FC fragment, followed by TSA. We suggest testing each approach in parallel to determine which conditions work best on a case-by-case basis. Most of our secondary antibodies, including both fluor- and HRP-conjugated, were obtained from Jackson ImmunoResearch Laboratories Inc., and are recommended for multilabeling experiments.

\section{Acknowledgments}

We thank members of the Krause lab for advice and support. Eric Lecuyer is supported by a fellowship from the Canadian Institutes of Health Research (CIHR). This technology was developed with support from the National Cancer Institute of Canada and CIHR.

\section{References}

1. Paddock, S. W., Langeland, A., DeVries, P. J., and Carroll, S. B. (1993) Threecolor immunofluorescence imaging of Drosophila embryos by laser scanning confocal microscopy. BioTechniques 14, 42-47.

2. Tautz, D. and Pfeiffle, C. (1989) A non-radioactive in situ hybridization method for the localization of specific RNAs in Drosophila embryos reveals translational control of the segmentation gene hunchback. Chromosoma 98, 81-85.

3. Hughes, S. and Krause, H. M. (1998) Double labeling with FISH in Drosophila whole-mount embryos. BioTechniques 24, 530-532.

4. Hughes, S. and Krause, H. M. (1998) Single and double FISH protocols for Drosophila, in Protocols in confocal microscopy, (Paddock, S., ed.), Humana, Totowa, NJ, pp. 93-101.

5. Raap, A. K., van de Corput, M. P., Vervenne, R. A., et al. (1995) Ultra-sensitive FISH using peroxidase-mediated deposition of biotin- or fluorochrome tyramides. Human Mol. Genetics 4, 529-534.

6. Wilkie, G. S. and Davis, I. (1998) Visualizing mRNA by in situ hybridization using high resolution and sensitive tyramide signal amplification. Elsevier Trends J., Technical Tips Online T01458.

7. Kosman, D., Mizutani, C. M., Lemons, D., Cox, W. G., McGinnis, W., and Bier, E. (2004) Multiplex detection of RNA expression in Drosophila embryos. Science 305, 846.

8. Tomancak, P., Beaton, A., Weiszmann, R., et al. (2002) Systematic determination of patterns of gene expression during Drosophila embryogenesis. Genome Biol. 3, Research 0088.1-0088.14.

9. Speel, E. J., Ramaekers, F. C., and Hopman, A. H. (1997) Sensitive multicolor fluorescence in situ hybridization using catalyzed reporter deposition (CARD) amplification. J. Histochem. Cytochem. 45, 1439-1446. 